\title{
Hysteretic nonequilibrium Ising-Bloch transition
}

\author{
Victor B. Taranenko, \\ Institute of Physics, National Academy of Sciences of the Ukraine, Kiev, Ukraine \\ Adolfo Esteban-Martín, Germán J. de Valcárcel, and Eugenio Roldán \\ Departament d'Òptica, Universitat de València, Dr. Moliner 50, 46100-Burjassot, Spain
}

Abstract

We show that a parametrically driven cubic-quintic complex Ginzburg-Landau equation exhibits a hysteretic nonequilibrium Ising-Bloch transition for large enough quintic nonlinearity. These results help to understand the recent experimental observation of this pheomenon [A. Esteban-Martín et al., Phys. Rev. Lett. 94, 223903 (2005)].

PACS numbers: 42.65.Sf, 47.54.+r,42.65.Hw

Spatially extended bistable systems with broken phase invariance display defects in the form of interfaces, socalled domail walls (DWs). A paradigm for the study of DWs is the parametrically driven complex GinzburgLandau equation, which can be written in the form [1]

$\partial_{t} A=\gamma A^{*}+(\mu+i \nu) A+(1+i \alpha) \partial_{x}^{2} A-(1+i \beta)|A|^{2} A$,

where $\gamma$ is the parametric pump, $\mu$ accounts for linear gain or loss, depending on its sign, $\nu$ is a detuning, $\alpha$ is the diffraction coefficient, and $\beta$ is the nonlinear dispersion coefficient. In writing Eq. (11) the spatial coordinate and the field amplitude have been normalized to the square root of the diffusion coefficient and of the saturation coefficient, respectively. This equation represents a universal description of parametrically excited waves [2] as well as of the close to threshold dynamics of self-oscillatory systems externally forced at the second harmonic of the natural oscillations frequency [1].

In Eq. (11) the phase invariance of the field $A(x, t)$ is broken because of the presence of the parametric term $\gamma A^{*}$, i.e, Eq. (11) shows the discrete symmetry $A \leftrightarrow-A$. This makes possible the existence of domain walls (DWs) that connect spatial regions where the field passes from, e.g., the homogeneous solution $A_{0}$ to the equivalent solution $-A_{0}$. There are two types of DWs, namely Ising and Bloch walls, which differ in the way the field crosses the complex zero at the DW core: In the Ising wall, both the real and the imaginary parts of the field become null, whilst in the Bloch wall the real and the imaginary parts become null at different spatial points. Then, in terms of the field intensity, $|A|^{2}$, an Ising wall is dark at its center whilst it is grey in the case of a Bloch wall. But the most striking difference between Ising and Bloch walls lies in their different dynamic behaviour: When nonvariational terms are present (in Eq. (11) this means that $\nu, \alpha$ or $\beta$ be different from zero) Bloch walls move whilst Ising walls remain at rest.

Coullet et al. 1] have discussed the above in detail for $\mu>0$ and have shown how Ising walls bifurcate into Bloch walls through the so-called nonequilibrium IsingBloch transition (NIBT), that takes its name from the equilibrium Ising-Bloch transition ocurring in ferromagnets [3]. Subsequently it was also shown that the NIBT occurs in Eq. (11) for negative $\mu$ [2], in which case DWs connect not only equivalent homogeneous solutions but also spatially modulated solutions.

There are a few experimental observations of this phenomenon. As far as we know, it has been reported only in liquid crystals [4] either subjected to rotating magnetic fields, [5, 6] or to an alternate electrical voltage [7]. This last experiment constitutes a particularly clear observation of the NIBT free from 2D effects, which complicate front dynamics through curvature effects. We must add our very recent observation of a hysteretic Ising-Bloch transition in a nonlinear optical cavity [8]. This last experiment was carried out in a photorefractive oscillator in a degenerate four-wave mixing configuration [8, 9, 10] and the cavity detuning played the role of the control parameter. We found that for small positive cavity detuning the system exhibits Ising walls. When detuning was increased, Ising walls bifurcated into Bloch walls at a cavity detuning value $\nu_{I B}$, and for $\nu>\nu_{I B}$ DWs were always of Bloch type. Then, when making a reverse detuning scan we found that Bloch walls existed up to a detunig value $\nu_{B I}$ where a new Ising-Bloch transition occurs. The interesting thing is that $\nu_{B I}<\nu_{I B}$, and then there is a detuning domain, $\nu_{B I}<\nu<\nu_{I B}$, where Ising and Bloch walls coexist.

The origin of the hysteresys was experimentally found to lie in the existence of bistability in the homogeneous state of the system: The homogeneous state exhibits bistability within a certain cavity detuning range between two spatially homogeneous states, say $A_{\text {hom, } 1}$ and $A_{\text {hom }, 2}$, with $\left|A_{\text {hom }, 1}\right|^{2}>\left|A_{\text {hom }, 2}\right|^{2}$. It occurs to happen that DWs connecting $A_{\text {hom, } 1}$ with $-A_{\text {hom, } 1}$ are of Ising type whilst those connecting $A_{\text {hom }, 2}$ with $-A_{\text {hom,2 }}$ are of Bloch type. Eq. (11) does not give any insight into this type of behaviour as the NIBT it exhibits is not hysteretic nor its homogeneous solution exhibits bistability of the type we are describing. Here we try to put some light into this problem by considering a straightforward generalization of Eq. (1). 
Hysteretic Ising-Bloch transitions have been theoretically described recently in two very different contexts. On the one hand, it has been described in the anisotropic $\mathrm{XY}$-spin system in an oscillatory magnetic field 11]. In that paper, Eq. (11) is studied for $v=\alpha=\beta=0$ plus an additional modulation term. As the system under study is variational, Bloch walls do not move and the hysteresys is found on the average oscillation period of the DW for certain parameter sets. On the other hand, hysteresis has also been found in the routes leading from standing fronts to a couple of counterpropagating ones in two discrete models (an array of Lorenz units and the FitzHugh-Nagumo model) in Ref. 12]. These previous theoretical results do not help to understand the experimental results in 8 that we have just resumed. As already outlined in [8] we will show that the addition of a quintic nonlinearity in Eq. (1) allows to understand qualitatively the experimental results.

We start with the following natural generalization of Eq. (1)

$$
\begin{aligned}
\partial_{t} A & =\gamma A^{*}+(\mu+i \nu) A+(1+i \alpha) \partial_{x}^{2} A \\
& -\left(1+i \beta_{3}\right)|A|^{2} A-\left(\alpha_{5}+i \beta_{5}\right)|A|^{4} A
\end{aligned}
$$

i.e., we have added a fifth order nonlinearity with $\alpha_{5}$ and $\beta_{5}$ the quintic saturation and nonlinear dispersion coefficientes, respectively. As we are interested in the minimal modification of Eq. (11) that contains a hysteretic NIBT, in the following we shall concentrate on the special case $\alpha_{5}=0$, as the addition of the quintic nonlinear dispersion term is enough for our purposes as we show next.

In Fig. 1 we represent the square modulus of the homogeneous solution of Eq. (2) as a function of detuning $\nu$ for $\gamma=2, \mu=\alpha=-\beta_{3}=1$ and the different values of $\beta_{5}$ indicated in the figure inset. Notice that there are two regions in which the homogeneous solution is multivalued: For negative detuning, where there is coexistence between two homogeneous solution values and the trivial solution, and also for positive detuning whenever $\beta_{5}>\beta_{5}^{c} \approx 0.39428$, where there is coexistence of three homogeneous solutions. We find that the latter requires that the signs of the nonlinear dispersion coefficients, $\beta_{3}$ and $\beta_{5}$ are different, and will concentrate on this case. Note that Eq. (2) holds the symmetry $\left(A, \nu, \alpha, \beta_{3}, \beta_{5}\right) \longleftrightarrow\left(A^{*},-\nu,-\alpha,-\beta_{3},-\beta_{5}\right)$ and consequently, the behaviour of the homogeneous solution is the same for the parameter sets $\left(\nu, \beta_{3}, \beta_{5}\right)$ and $\left(-\nu,-\beta_{3},-\beta_{5}\right)$.

The numerical integration of Eq. (2) shows that for negative detuning $\nu$, the system exhibits extended patterns and that the homogeneous solution can be stable for positive $\nu$. Then it is for $\nu>0$ that we can find DWs connecting homogeneous solutions and we concentrate in this case (Notice that for $\beta_{5}=0$ this is the parameter region, $\nu>0$, where the NIBT was studied in [1] and 2]).
We have carried out the numerical integration of Eq. (2) for $\gamma=2, \mu=\alpha=1$ and different values of $\beta_{3}$ and $\beta_{5}$. We pass to comment first our results for $\beta_{3}=-1$ and different values of $\beta_{5}$ and $\nu$.

In Fig. 2 we represent again the homogeneous steady state for the same parameters as in Fig. 1 (except for the values of $\beta_{5}$ ), and have marked the different patterns one can observe. For $\beta_{5}=0.38<\beta_{5}^{c}$, Fig. $2(\mathrm{a})$, the homogeneous solution is single-valued and two types of DWs are observed: Ising walls ( $I W$ in the figure), for detunings $\nu \leq 1.24$; and Bloch walls $(B W)$ for $\nu>1.24$. For $\nu>1.53$ the homogeneous solution becomes modulationally unstable; Bloch walls connect patterns in this region. In Fig. 3 both the intensity and phase spatial profiles corresponding to an Ising wall, Fig. 3(a), and a Bloch wall, Fig. 3(b), are represented. Notice that the field intensity is null at the DW core only in the Ising wall, and that the phase jump is sharp (smooth) for the Ising (Bloch) wall. It is also interesting to notice the shoulder in the field intensity in the case of the Bloch wall (the shoulder appears on the back side of the wall with respect to the direction of movement).

As the value of $\beta_{5}$ is increased, new features appear. For $\beta_{5}=0.395$ (i.e., slightly larger that $\beta_{5}^{c}$ ) the homogeneous solution becomes multivalued, Fig. 2(b) (the dashed line indicates that the homogeneous solution is unstable). In this case Ising walls do not bifurcate directly into moving Bloch walls, but start oscillating periodically around a fixed position, that is, there is not a net displacement of the wall. Fig. 4 shows the intensity and phase profiles of the oscillating wall in three different instants of time and it can be appreciated how the wall pases from a clear Bloch character (smooth phase jump) when it is at the center of the oscillation, Fig. 4(b), to a clear Ising character (sharp phase jump) when it is at the extremes of the oscillation, Figs. 4(a) and (c).

The behaviour just described appears when $\beta_{5} \simeq \beta_{5}^{c}$. When $\beta_{5}$ is further increased a new and remarkabe effect appears: There is a detuning range of coexistence between the oscillating and the Bloch walls, see Fig. 2(c). This is better appreciated in Fig. 5 where the velocity of the walls is represented as a function of detuning for the same parameters as in Fig. 2. In Figs. 5(a) and 5(b) the behaviour of the velocity closely follows that of the standard NIBT 1, 2], but in Fig. 5(c) the new phenomenon of the hysteretic NIBT is clearly appreciated.

In the case we have described no coexistence between Ising and Bloch walls is observed, only between oscillating and Bloch walls. But by decreasing the value of $\beta_{3}$ from -1 to -1.5 we can observe this coexistence. In Fig. 6 we represent again the wall velocity as a function of detuning for the same values as in Fig. 2 except $\beta_{3}=-1.5$ and $\beta_{5}=0.6$. The behaviour is similar to that described above but now there appears a wide domain of coexistence between Bloch walls and both oscillating and Ising walls. This is a much clearer hysteretic NIBT. 
These results show that the addition of a quintic nonlinear dispersion term to the parametrically driven complex Ginzburg-Landau equation suffices for obtaining a hysteretic nonequilibrium Ising-Bloch transition (HNIBT), a phenomenon first observed in 8]. As a fifthorder nonlinearity represents the simplest, higher order correction to the usual complex Ginzburg-Landau, and the latter is of wide applicability in physical and chemical systems, the HNIBT could be well observed in other systems. While we do not claim that this simple model, Eq. (2), represents an accurate description of the experimental system in [8] (the photorefractive nonlinearity is saturating) we note that it describes qualitatively the experimental observations, even reproducing small details such as the shoulder in the field intensity in the case of the Bloch wall, Fig. (3), that has been repeatidly observed during the experiments in [8].

This work has been supported by Spanish Ministerio de Educación y Ciencia and European Union FEDER through projects BFM2002-04369-C04-01.

[1] P. Coullet, J. Lega, B. Houchmanzadeh and J. Lajzerowicz, Phys. Rev. Lett. 65, 1352 (1990).

[2] G. J. de Valcárcel, I. Pérez-Arjona and E. Roldán, Phys. Rev. Lett. 89, 164101 (2002).

[3] L. N. Bulaevskii and V. L. Ginzburg, Zh. Eksp. Teor. Fiz. 45, 772 (1963) [Sov. Phys. JETP 18, 530 (1964)].

[4] E. Meron, DDNS 4, 217 (2000).

[5] T. Frisch, S. Rica, P. Coullet, and J. M. Gilli, Phys. Rev. Lett. 72, 1471 (1994).

[6] S. Nasuno, N. Yoshimo, and S. Kai, Phys. Rev. E 51, 1598 (1995).

[7] T. Kawagishi, T. Mizuguchi, and M. Sano, Phys. Rev. Lett. 75, 3768 (1995).

[8] A. Esteban-Martín, V. B. Taranenko, J. García, G. J. de Valcárcel, and E. Roldán, Phys. Rev. Lett. 94, 223903
(2005).

[9] Ye. Larionova, U. Peschel, A. Esteban-Martín, J. García Monreal, and C. O. Weiss, Phys. Rev. A 69, 033803 (2004).

[10] A. Esteban-Martín, J. García, E. Roldán, V. B. Taranenko, G. J. de Valcárcel, and C. O. Weiss, Phys. Rev A 69, 033816 (2004).

[11] N. Fujiwara, H. Tutu, and H. Fujisaka, Phys. Rev. E 70, 066132 (2004).

[12] D. Pazó, R.R. Deza, and V. Pérez-Muñuzuri, Phys. Lett. A 340, 132 (2005).

\section{Figure Captions}

Fig. 1. Homogeneous solution intensity as a function of detuning for $\gamma=2, \mu=\alpha=-\beta_{3}=1$ and $\beta_{5}=0.35$ (i), $\beta_{5}=0.3928$ (ii), and $\beta_{5}=0.45$ (iii), which is an enlargement of a part of the positive detuning domain.

Fig. 2. Homogeneous solution intensity for the same parameter values as Fig. 1 except $\beta_{5}$, which are marked in the figure. The different pattern domains are marked as $I W$ (Ising walls), $B W$ (Bloch walls), $O W$ (oscillating walls), and $P$ (patterns). The continuous (dashed) line indicates stable (unstable) homogeneous solution.

Fig. 3. Intensity (full line) and phase (dashed line) spatial profiles of an Ising (a) and a Bloch (b) wall for the same parameters as Fig. 2(a) and the detuning values marked in the figure.

Fig. 4. Intensity (full line) and phase (dashed line) profiles of an oscillating wall at three different instants of time (see text). The parameters are the same as in Fig. $2(\mathrm{~b})$ and $\nu=1.29$.

Fig. 5. Velocity of the domain walls as a funtion of detuning for the same parameter values as in Fig. 2. The gray areas mark the velocitie of the oscillating walls. The arrows mark the transition from Bloch walls to oscillating walls when a decreasing detuning scan is carried out.

Fig. 6. Velocity of the domain walls as a function of detuning for $\gamma=2, \mu=\alpha=1, \beta_{3}=-1.5$, and $\beta_{5}=0.6$. 


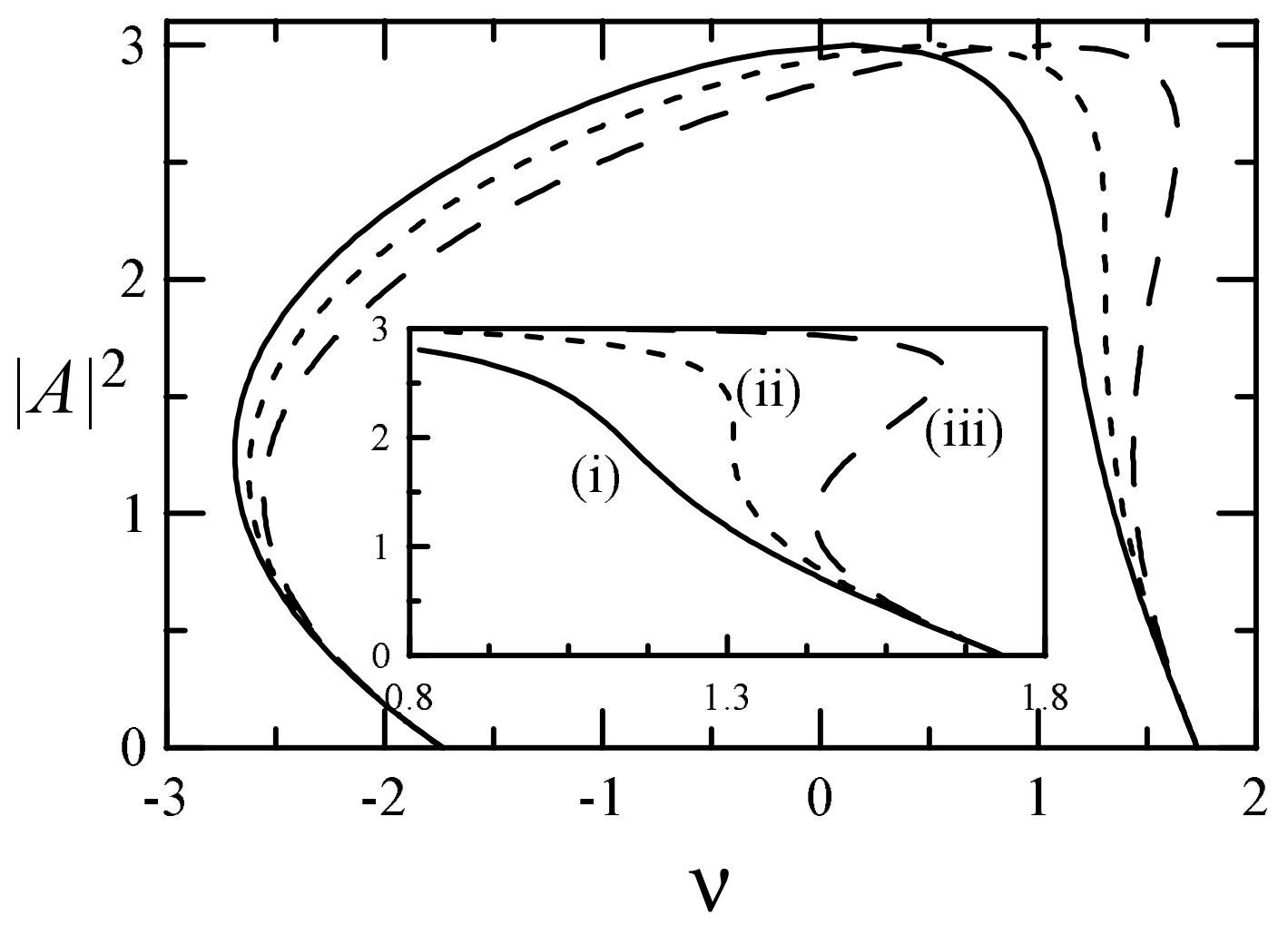




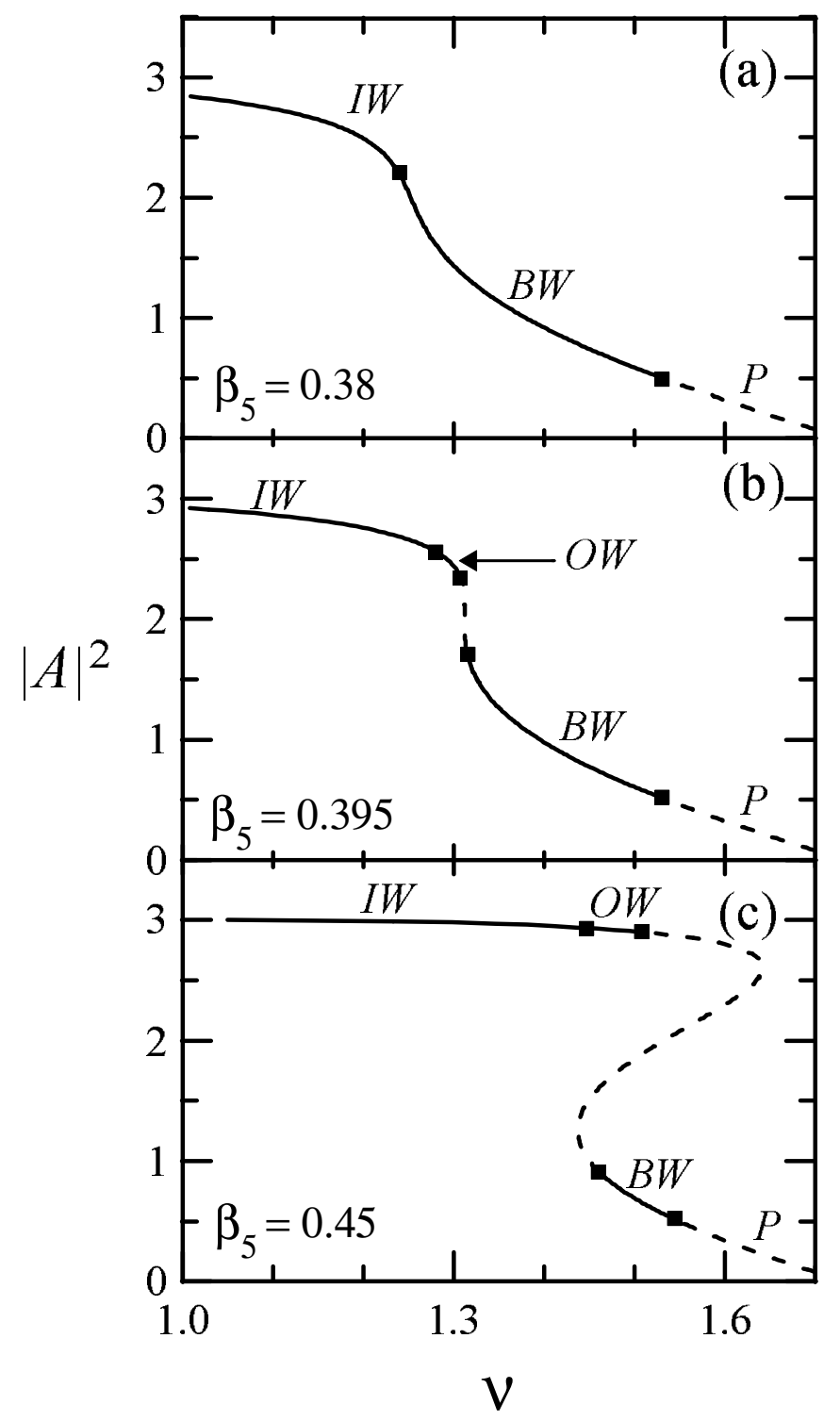




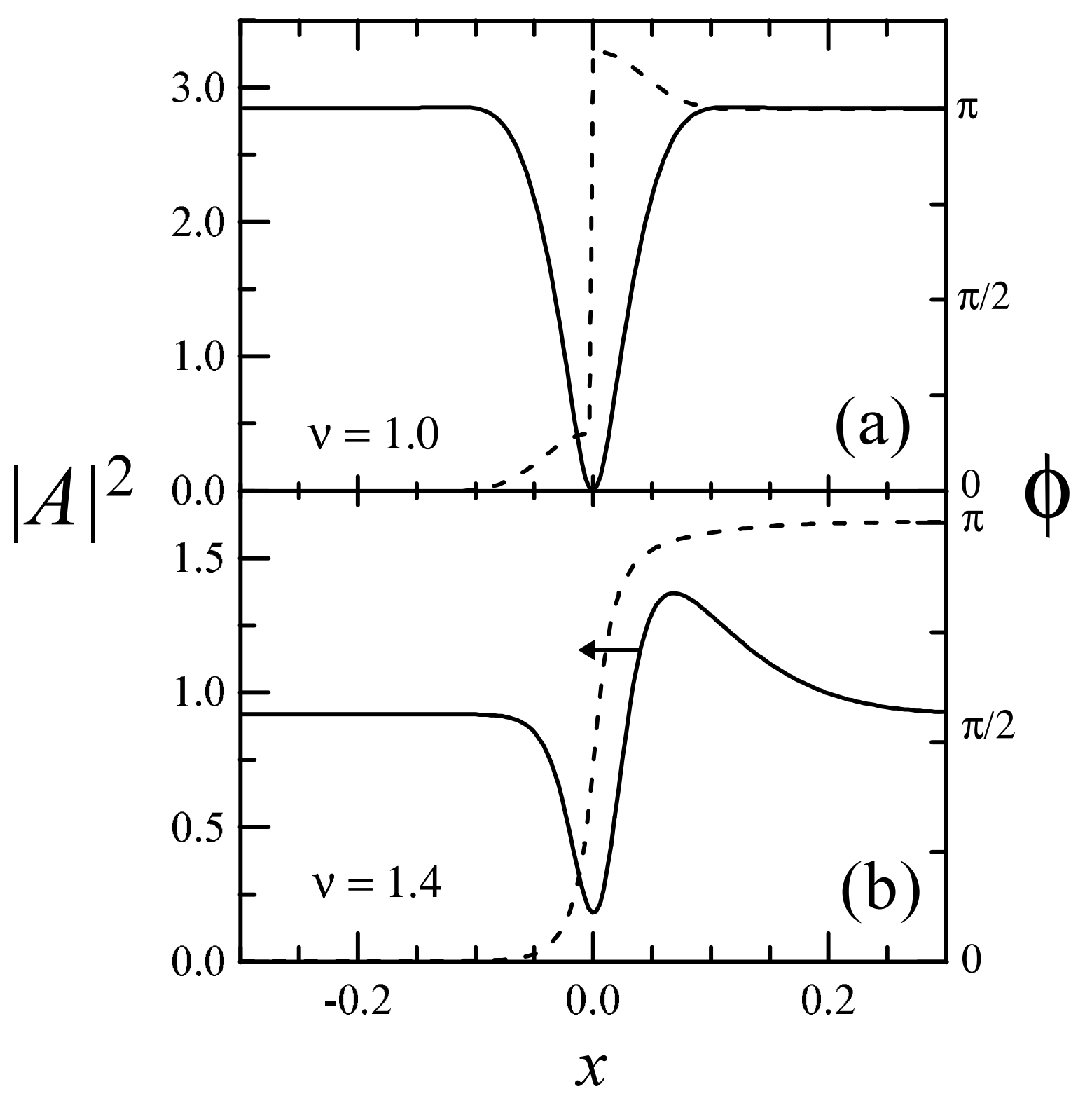




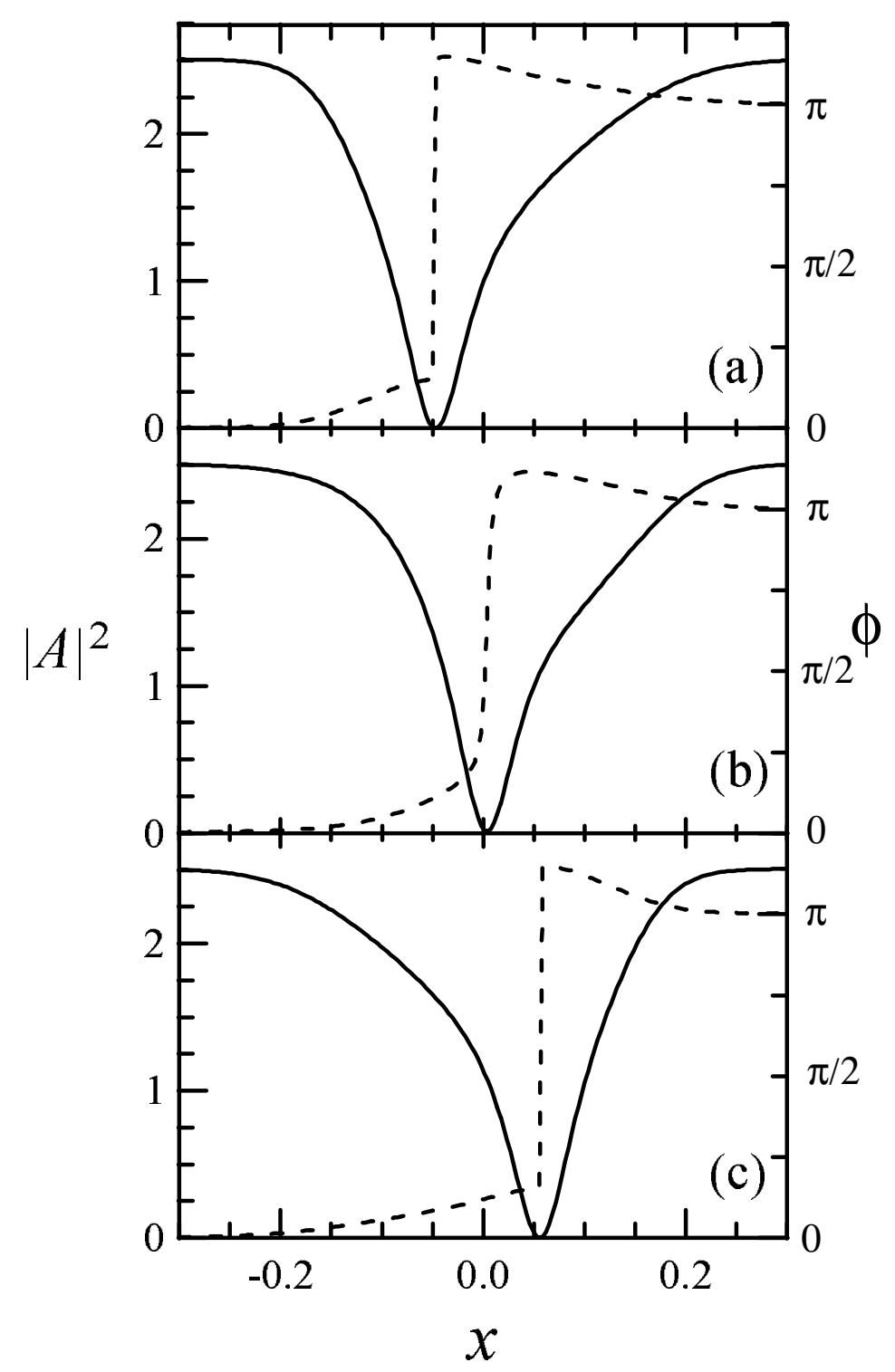




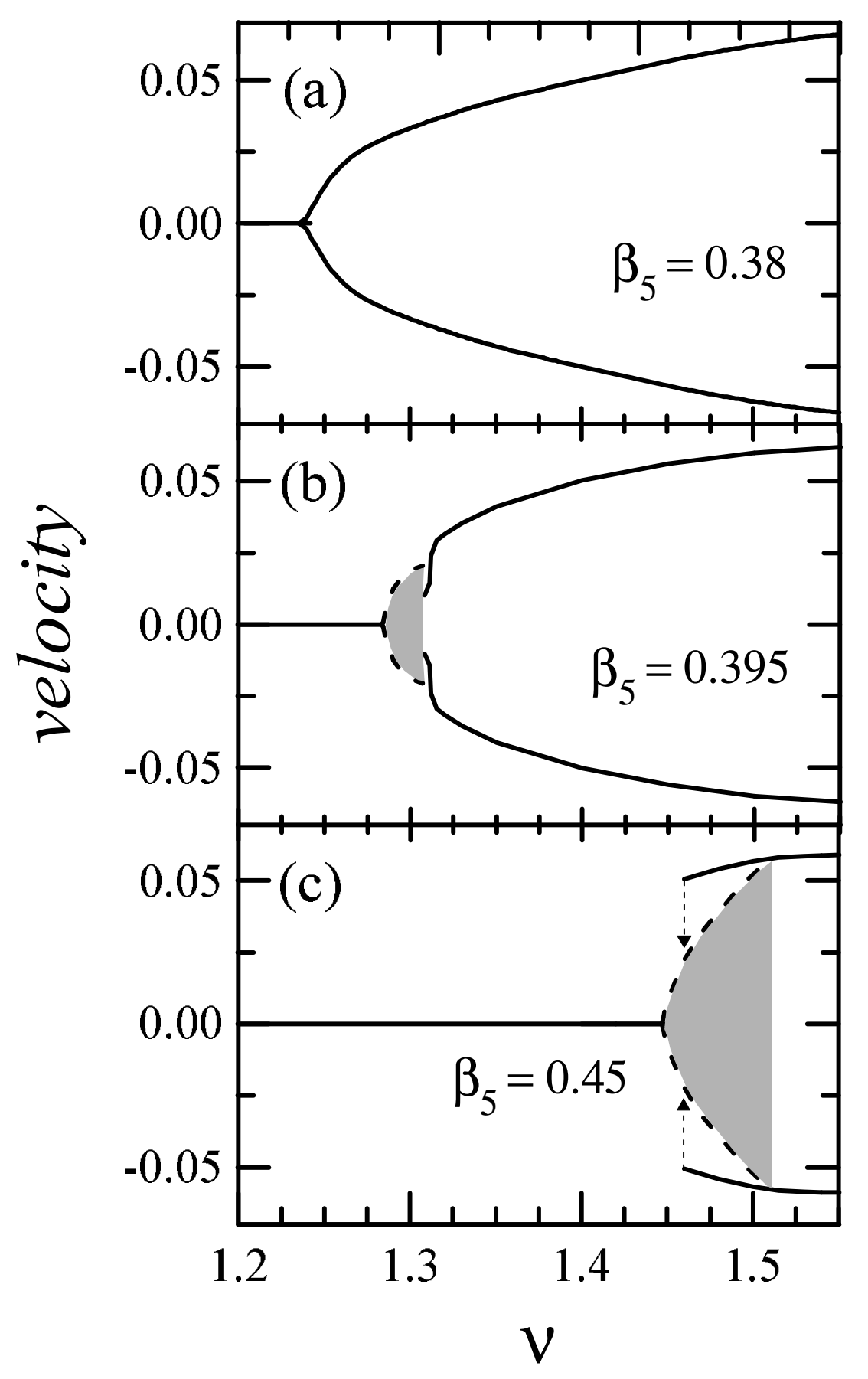




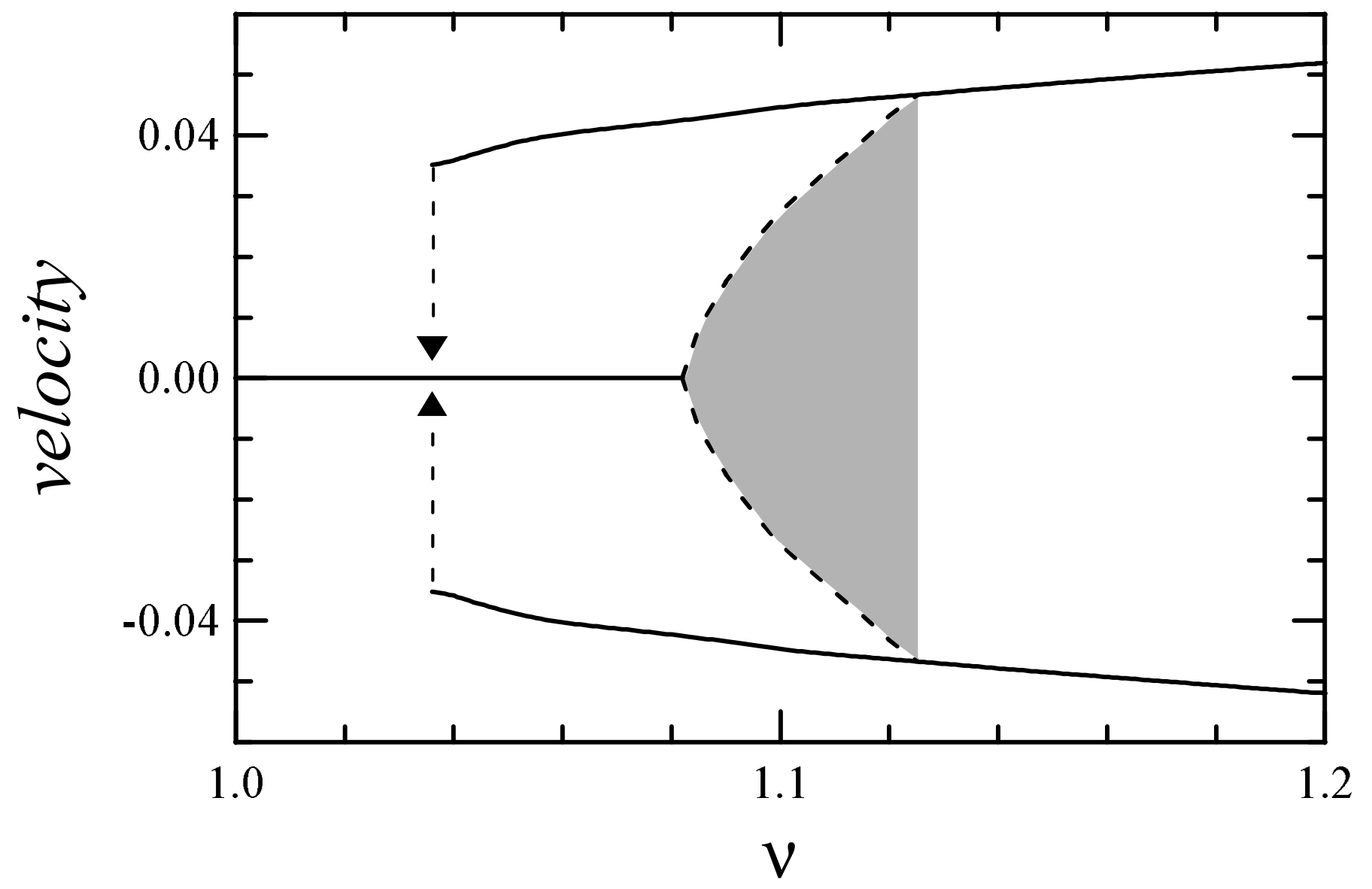

